\title{
Evaluation of Lipid Peroxide Metabolism in Neonatal Rats during Suckling: Developmental Changes in Whole Body Lipid Peroxides
}

\author{
Sachie TAtsumi, ${ }^{1}$ Shinichi SAItoH, ${ }^{2}$ and Masashige SuzUKi, ${ }^{2, *}$ \\ ${ }^{1}$ Laboratory of Food and Nutrition, Department of the Science of Living, \\ Kyoritsu Women's Junior College, \\ Chiyoda-ku, Tokyo 101, Japan \\ ${ }^{2}$ Laboratory of Biochemistry of Exercise and Nutrition, \\ Institute of Health and Sport Sciences, \\ University of Tsukuba, Tsukuba 305, Japan
}

(Received June 29, 1992)

\begin{abstract}
Summary To evaluate lipid peroxide metabolism in neonatal rats during suckling, we studied developmental changes in whole body lipid peroxidation by measurement of thiobarbituric acid-reactive substances (TBARS) determined as malondialdehyde. Whole body TBARS contents were very low immediately after birth but increased sharply at 3-9 days, peaked at 9-15 days, and then remained unchanged until 21 days. The feeding of a skimmed milk diet to neonates between 17 and 20 days old sharply decreased their whole body TBARS contents at 20 days as compared with those at 16 days, however, this was not observed by the feeding of an artificial milk diet containing $3 \mathrm{~g}$ of soybean oil/100 $\mathrm{g}$ diet. In vitro ethane production by liver and brain homogenates and in vivo breath ethane levels in expired air were higher at 20 or 21 days than at 10 days after birth. These results suggest that lipid peroxide formation in neonatal rats increases shortly after birth and that its degradation and/or excretion may increase during the latter period of suckling.
\end{abstract}

Key Words: lipid peroxides, TBARS, whole body, ethane production, neonatal rat

Lipid peroxidation of biological membranes is considered as a basic deteriorative reaction in the cellular mechanisms of aging [1]. Because lipid peroxidation has been associated with oxygen toxicity and the aging process, studies have been

* To whom correspondence should be addressed. 
initiated to determine the extent of lipid peroxide formation in animals of various ages $[2,3]$.

It has been reported [3] that liver lipid peroxidation by-products, thiobarbituric acid-reactive substances (TBARS), were extensively high in rat fetuses and early newborns at about 10-15 days after birth, and thereafter decreased sharply. These trends were associated with a remarkable increase in superoxide dismutase (SOD) and catalase activities in the liver: the levels of these enzyme activities were low at birth and increased rapidly after the 10th day [3]. However, glutathione peroxidase (GSH-Px) activity in the liver increased only slightly [3, 4]. In contrast, lung TBARS contents were not detectable in rats immediately after birth but appeared by the second day, reached a maximum at 5 days, and then gradually disappeared by 20 to 21 days after birth [2]. It has been reported [5] that maturation of the lung antioxidation enzyme system is well complete before birth.

Glutathione-S-transferase (GSH-tr) activity in rat brain was low at 7 days, but gradually increased 2.5-fold over the age of 21 days [6]. Blood TBARS contents were low in the fetus and early newborn period, then increased and reached a peak at 15 days, and remained unchanged until 21 days of age [3]. Serum GSH-tr in rats progressively increased from immediately after birth and reached maximum at 140 days of age [7]. From these results, it has been suggested that developmental changes in lipid peroxidation are different from tissue to tissue and that a high content of the lipid peroxidation by-product TBARS in some tissues did not always coincide with high activity of antioxidation enzymes in the tissue.

As the above-mentioned studies [2-7] have shown, most of lipid antioxidation-related enzyme activities in serum and tissues of newborn and neonatal animals increase during the latter half of the suckling period, and degradation and/or excretion capacities of whole body lipid peroxides of neonatal rats may develop until 21 days after birth. However, to our knowledge, this possibility has not been yet examined by the measurement of whole body lipid peroxides. We previously reported [8] that more than $80 \%$ of the total TBARS content in the rat body is distributed in the carcass portion. Furthermore, we observed [9] that the whole body TBARS content of 10-day-old rats was significantly higher than that of 21-day-old ones.

In the study reported herein, we examined developmental changes in whole body lipid peroxidation in neonatal rats during the suckling period by examining whole body TBARS contents, ethane production by liver and brain homogenates, and breath ethane levels. In addition, we also tested whether degradation and/or excretion capacities of whole body lipid peroxides might increase during the latter half of suckling by the feeding of an artificial milk diet.

\section{METHODS}

Four experiments were conducted using Wistar rats (CLEA Japan Inc., Tokyo; and Doken Inc., Shimodate, Japan). Animals were housed in a tempera- 
ture-controlled room $\left(23-24^{\circ} \mathrm{C}\right)$ with a 12 -h light-12-h dark cycle. Rats were given a commercial diet (NMF, Oriental Yeast Co., Ltd., Tokyo) in each experiment. Water was provided freely.

Experiment 1. Eight and 5 virgin rats of 10-12 weeks of age were respectively mated in experiments $1-\mathrm{A}$ and $1-\mathrm{B}$ and given NMF throughout the periods of gestation and lactation. In experiment 1-A, one dam was randomly selected from 8 dams and subsequently all pups from the dam were sacrificed at 1 day of age, and remaining litters of the $7 \mathrm{dams}$ were breast-fed by the respective dams. Then, 1 litter from the remaining litters was randomly selected and sacrificed at each of the following ages: $3,6,9,12,15,18$, and 21 days. In experiment 1-B, 4-5 pups of each litter of the 5 dams were sacrificed at birth. Remaining littermates were culled to 5 pups per litter, breast-fed for 14 days, and then sacrificed. All pups were sacrificed under ethylether anesthesia, and their whole bodies were stocked in nitrogen gas streamed bottles at $-30^{\circ} \mathrm{C}$ until the determination of whole body lipid peroxides.

Experiment 2. Four virgin female rats of 12 weeks of age were mated and given the NMF diet for the periods of gestation and lactation. At birth all litters were culled to 10 pups per litter. Two pups from each litter were randomly selected and sacrificed at 10 days of age. Two pups from each litter were also randomly selected and sacrificed at 20 days of age. The pups were sacrificed under ethylether anesthesia, and their brain and liver were removed, homogenized, and used for assessment of in vitro ethane production.

Experiment 3. Three virgin female rats of 12 weeks of age were mated and given NMF for the periods of gestation and lactation. At birth all litters were culled to four pups per litter and their breath was measured for its ethane content at 10 and 21 days of age.

Experiment 4. Four virgin female rats of 10-12 weeks of age were mated and given NMF for the periods of gestation and the first 16 days of lactation. On day $16,7-8$ pups from each litter were randomly selected and sacrificed. The remaining 6 pups from each litter were divided into 2 equal groups. One group was given an artificial milk diet; and the other a skimmed milk diet for 4 days. The two groups of pups were individually housed in small cages and allowed free access to the milk diets. About $75 \%$ of pups in each group were successfully accustomed to the feeding of milk diets. These pups were sacrificed at 20 days of age under ethylether anesthesia, and their whole bodies were stocked as described in experiment 1 . The milk diet consumption of the pups was measured at 8-h intervals during the 4-day feeding of the milk diet in terms of the change in body weight.

Analyses. Whole body and tissue lipid peroxides, defined as TBARS, were determined by the method of Uchiyama and Mihara [10]. In vitro ethane production by tissue homogenates was measured mostly by the method of Riely and Cohen [11]. Fresh samples of brain and liver were homogenized in $0.4 \mathrm{~mm}$ butylated hydroxytoluene [12] containing $0.05 \mathrm{M}$ phosphate buffer in saline $(\mathrm{pH}$ 7.4) $[13]$. The homogenates were incubated in a tube capped with a double 
gum-stopper at $37^{\circ} \mathrm{C}$ for $60 \mathrm{~min}$ under gentle shaking. Ethane production by the homogenates was determined by gas-chromatographic measurement of the gas content in air samples obtained from the top space of the incubation tube. The apparatus used was a gas-chromatograph (JGC-1100, JEOL Co., Ltd., Tokyo) equipped with a stainless column ( $3 \mathrm{~mm}$ in diameter, $2 \mathrm{~m}$ in length) packed with $\mathrm{SiO}_{2}$-Unibeads 2S (Gaschro-Kogyo Inc., Tokyo). Breath ethane content was measured by the method of Hafeman and Hoekstra [14]. Four pups from each litter were sealed into a 1.5-liter vacuum desiccator in an air environment. Initial air composition and pressure were maintained by addition of oxygen from an external oxygen supply at the rate at which it was consumed by the rats. Chamber air was cycled by an air pump. Ammonia and carbon dioxide were removed from the chamber by passage of the air through $30 \% \mathrm{H}_{2} \mathrm{SO}_{4}$ and $5 \mathrm{~N}-\mathrm{NaOH}$, respectively.

One-milliliter samples were taken from the sampling port $2 \mathrm{~h}$ after the rats had been placed in the chamber, and ethane production was determined by the gas chromatograph.

Statistics. Results are expressed as mean \pm SEM. Data were analyzed by one-way analysis of variance (ANOVA). Significant differences between means were determined by use of Tukey's multiple comparison. Differences were considered to be significant if $p$ values of $<0.05$ were obtained.

\section{RESULTS}

Developmental change in whole body TBARS content in neonatal rats during suckling (experiments $1-A$ and $1-B$ )

In experiment $1-\mathrm{A}$, the whole body TBARS content in neonatal rats was very low immediately and 2-3 days after birth, increased sharply at 3-9 days, peaked on about 9-15 days, and remained unchanged until 21 days of age (Fig. 1).

Experiment 1-B was conducted to re-confirm the remarkable increase in whole body TBARS content during the first 2 weeks of the suckling period. Whole body TBARS contents of rats at 14 days of age were significantly higher than those at birth (14 days vs. 1 day: $29.3 \pm 2.7$ vs. $0.1 \pm 0.0 \mu$ mol MDA/pup, $p<0.05$ ).

Changes in ethane production by liver and brain homogenates in neonatal rats during the suckling period (experiment 2)

Ethane production by liver homogenates was significantly higher at 20 days than at 10 days of age, and this also held true for the brain homogenate (Table 1).

Changes in ethane content of breath of neonatal rats during the suckling period (experiment 3)

No ethane was detected in the expired air of the pups at 10 days after birth; however, at 21 days thereafter the concentration of the gas was $415-922 \mathrm{pmol} / \mathrm{ml}$ of chamber air/4 pups. 


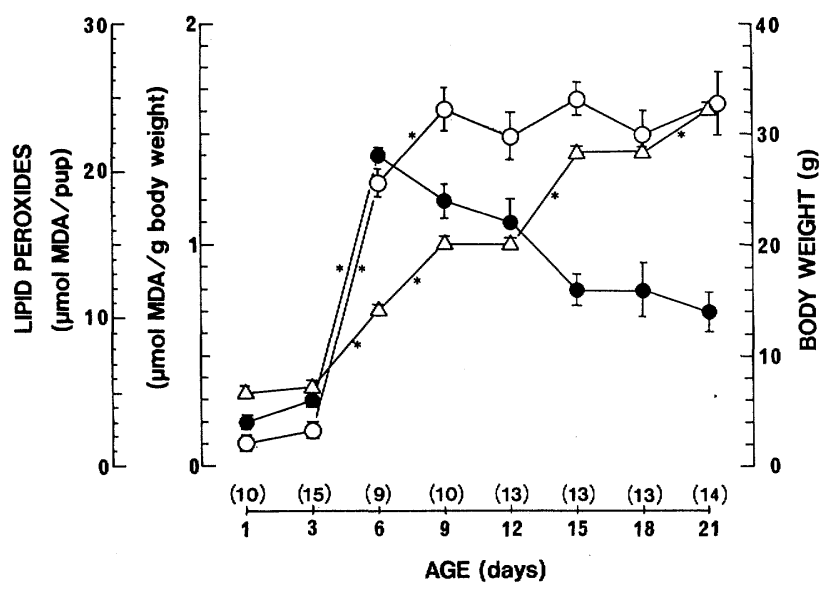

Fig. 1. Developmental change in whole body TBARS content in neonatal rats during the suckling period (experiment 1-A). Mean value of TBARS contents in NMF was 20.7 $\mu \mathrm{mol} \mathrm{MDA} / 100 \mathrm{~g}$ diet [9]. $\bigcirc \mathrm{O}$, lipid peroxides ( $\mu$ mol MDA/pup); $\bullet \bullet$, lipid peroxides $(\mu \mathrm{mol} \mathrm{MDA} / \mathrm{g}$ body weight); $\triangle-\triangle$, body weight $(\mathrm{g})$. Vertical lines represent \pm 1 SEM; number of pups in parentheses. * Significant difference between the two values of adjacent points $(p<0.05)$.

Table 1. Changes in in vitro ethane production by liver and brain homogenates in neonatal rats (experiment 2). ${ }^{\mathrm{a}}$

\begin{tabular}{lcc}
\hline & \multicolumn{2}{c}{ Age (days) } \\
\cline { 2 - 3 } & 10 & 20 \\
\hline Number of pups (n) & $(8)$ & $(8)$ \\
Body weight (g) & $19.8 \pm 0.8$ & $44.8 \pm 2.6^{\mathrm{b}}$ \\
Liver weight (mg) & $500 \pm 20$ & $990 \pm 40^{\mathrm{b}}$ \\
Brain weight (mg) & $990 \pm 20$ & $1,277 \pm 19^{\mathrm{b}}$ \\
Ethane production & & \\
Liver: (pmol ethane/liver) & $224 \pm 40$ & $772 \pm 92^{\mathrm{b}}$ \\
(pmol ethane/g liver) & $445 \pm 82$ & $772 \pm 87^{\mathrm{b}}$ \\
Brain: (pmol ethane/brain) & $396 \pm 101$ & $778 \pm 143^{\mathrm{b}}$ \\
(pmol ethane/g brain) & $400 \pm 99$ & $609 \pm 112$ \\
\hline
\end{tabular}

${ }^{\text {a }}$ Values are mean \pm SEM. ${ }^{\mathrm{b}}$ Significantly different from corresponding value for 10 -day-old rats $(p<0.05)$.

Effect of an artificial milk diet or a skimmed milk diet for the last 4 days of the suckling period on whole body lipid peroxides in neonatal rats (experiment 4)

Body weights of the pups fed the artificial milk diet from 17 to 20 days of age showed a significant increase as compared with those at 16 days of age $(p<0.05)$ (Table 2). Although the diet consumption for 4 days was greater in the pups fed the skimmed milk diet than in those fed the artificial milk diet, body weights of the pups fed the skimmed milk diet were significantly lower than those of the pups fed the artificial milk diet. Whole body TBARS contents slightly increased in the 
Table 2. Effects of a skimmed milk diet (A) or an artificial rat milk diet (B) on whole body TBARS content in neonatal rats (experiment 4 ). ${ }^{\text {a }}$

\begin{tabular}{lcccccc}
\hline Diet & Age & & $\begin{array}{c}\text { Diet } \\
\text { consumption }\end{array}$ & $\begin{array}{c}\text { Body } \\
\text { weight }\end{array}$ & \multicolumn{2}{c}{ TBARS } \\
\hline & & & & & \multicolumn{2}{c}{$(\mu$ mol MDA $)$} \\
& $(\mathrm{d})$ & $(\mathrm{n})$ & $(\mathrm{g} / 4$ days $)$ & $(\mathrm{g})$ & $(/ \mathrm{pup})$ & $(/ \mathrm{g}$ pup $)$ \\
$\mathrm{A}^{\mathrm{b}}$ & 16 & 30 & & $31.4 \pm 1.9$ & $20.4 \pm 4.4$ & $0.65 \pm 0.01$ \\
$\mathrm{~B}^{\mathrm{c}}$ & 20 & 9 & $66.0 \pm 5.5$ & $34.5 \pm 0.6$ & $6.1 \pm 1.6^{\mathrm{d}}$ & $0.18 \pm 0.05^{\mathrm{d}}$ \\
\hline
\end{tabular}

${ }^{\text {a }}$ Values are mean \pm SEM. ${ }^{\mathrm{b}}$ Skimmed milk was purchased from Yukijirushi Food Industries Co., Tokyo. The composition of skimmed milk diet in percentages was as follows: skimmed milk, 20; sugar, 3; water, 77. ${ }^{c}$ The composition of the artificial milk diet was skimmed milk, 20; soybean oil, 3; water, 77. Fatty acid composition of the soybean oil was as follows (g/ $\mathrm{kg}): 16: 0,111 ; 18: 0,38 ; 18: 1,282 ; 18: 2,503 ; 18: 3,62$; and other constituents, 4 . ${ }^{\mathrm{d}}$ Significantly different from corresponding value for 16 -day-old pups $(p<0.05)$. ${ }^{\mathrm{e}}$ Significantly different from skimmed milk diet-fed group $(p<0.05)$.

pups fed the artificial milk diet, but significantly decreased in the pups fed the skimmed milk diet, resulting in a remarkable difference between the two groups.

\section{DISCUSSION}

In the present study, we measured whole body TBARS contents, and we assumed that the changes in those levels might reflect overall changes of lipid peroxidation in the body.

As mentioned previously, the results of earlier studies $[2-4,6,7]$ suggest that lipid peroxide contents and maturation of the antioxidation enzyme system in the body of neonatal rats are different from tissue to tissue and that it is difficult to identify the tissue(s) or enzyme(s) that might mainly contribute to the increase or decrease in whole body lipid peroxides in neonatal rats.

From the results of the present study, whole body TBARS contents were very low at birth, increased sharply within the first 2 weeks of age, and remained constant throughout the suckling period. These trends were also confirmed by the findings on in vivo and in vitro ethane production, which is considered to be an index of lipid peroxidation $[1,11]$. However, these observations do not agree with our previous study [9], where whole body TBARS contents of 10-day-old animals were significantly higher than those of 21-day-old ones. Because litter size was not adjusted in experiment 1-A, a possible effect of milk consumption on body growth may have caused the differences between both studies. Indeed, body weight of 21-day-old pups was heavier in the previous study than in the present one (42-55 vs. $32 \mathrm{~g}$ ); and when whole body TBARS content was expressed per g body weight (Fig. 1), the value at 9 days after birth was significantly higher than that at 21 days $(1.2 \pm 0.08$ vs. $0.7 \pm 0.09 \mu \mathrm{mol} \mathrm{MDA} / \mathrm{g}$ bw,$p<0.01)$.

Although the total lipid and its fatty acid composition in rat dam's milk [15] are quite different from the artificial milk diet used in the present study, whole 
body TBARS contents were not significantly affected by the artificial milk diet for the last 4 days of the suckling period. The lipid peroxide level in the present artificial milk diet $(19.6 \mathrm{nmol} \mathrm{MDA} / \mathrm{ml})$ was not significantly different from that in the milk from rat dams $(20.8-24.0 \mathrm{nmol} \mathrm{MDA} / \mathrm{ml})$ [9], but was higher than that in the skimmed milk diet (trace). These results suggest that the increase in whole body TBARS content during the early period of suckling was partly related to a possible increase in milk consumption in newborn rats. Because of the very low lipid content in the skimmed milk diet, the capacity of the body to form lipid peroxides was remarkably decreased in the pups fed the skimmed milk diet. And whole body lipid peroxides in the pups fed the skimmed milk diet would also decrease if their degradation and/or excretion capacities increased extensively.

Hydrocarbon gases can pass through the lungs into the expired air; therefore, breath ethane might be also considered an index of degradation and/or excretion of lipid peroxides [16]. In the present study, breath ethane production was remarkably high at 21 days after birth. Tanswell and Freeman [5] suggested that maturation of the pulmonary antioxidant enzyme system in rats is virtually complete before birth. Hence, degradation and/or excretion capacity of lipid peroxides other than lungs in the body of the pups might be increased by the end of the suckling period.

In the previous study, we reported [9] that the whole body TBARS content in newborn pups was very low. This was also observed in the present study. Since it has been reported $[17,18]$ that SOD activity in the human placenta increases during the latter half of pregnancy, the placenta may work as a barrier for transfer of lipid peroxides from dam to fetus.

In the present study, sex difference was not considered. A big sex difference is known to exist in rats in the capacity of their lipid antioxidation system, but this difference does not manifest itself until sexual maturity $[4,19]$.

From the results of the present study, we conclude that lipid peroxide formation in neonatal rats increases shortly after birth, and is followed by an increase in degradation and/or excretion of the peroxides during the latter period of suckling.

The authors wish to thank Mr. Y. Murata and Mr. K. Yamamoto for their assistance in ethane determinations.

\section{REFERENCES}

1. Sagai, M., and Ichinose, T. (1980): Age-related changes in lipid peroxidation as measured by ethane, ethylene, butane and pentane in respired gases of rats. Life Sci. 27, 731-738.

2. Kehrer, J.P., and Autor, A.P. (1977): Age-dependent lipid peroxidation in neonatal rat lung tissue. Arch. Biochem. Biophys., 181, 73-81.

3. Yoshioka, T., Takehara, Y., Shimatani, M., Abe, K., and Utsumi, K. (1982): Lipid peroxidation and antioxidants in rat liver during development. Tohoku J. Exp. Med., 137, 391-400.

4. Pinto, R.E., and Bartley, W. (1969): The effect of age and sex on glutathione reductase and

Vol. 14, No. 1, 1993 
glutathione peroxidase activities and on aerobic glutathione oxidation in rat liver homogenates. Biochem. J., 112, 109-115.

5. Tanswell, A.K., and Freeman, A.B. (1984): Pulmonary antioxidant enzyme maturation in the fetal and neonatal rat. I. Developmental profiles. Pediatr. Res., 18, 584-587.

6. Das, M., Dixit, R., Seth, P.K., and Mukhtar, H. (1981): Glutathione-S-transferase activity in the brain: Species, sex, regional, and age differences. J. Neurochem., 36, 1439-1442.

7. Mukhtar, H., and Bend, J.R. (1977): Serum glutathione S-transferases: Perinatal development, sex difference, and effect of carbon tetrachloride administration on enzyme activity in the rat. Life Sci, 21, 1277-1286.

8. Suzuki, M., Katamine, S., and Tatsumi, S. (1983): Exercise-induced enhancement of lipid peroxide metabolism in tissues and their transference into the brain in rat. J. Nutr. Sci. Vitaminol., 29, 141-151.

9. Tatsumi, S., Saitoh, S., and Suzuki, M. (1992): Intake of air-oxidized soybean oil by dams does not affect whole body lipid peroxide contents of their newborn and suckling pups. $J$. Clin. Biochem. Nutr., 12, 77-83.

10. Uchiyama, M., and Mihara, M. (1978): Determination of malonaldehyde precursor in tissues by thiobarbituric acid test. Anal. Biochem., 86, 271-278.

11. Riely, C.A., and Cohen, G. (1974): Ethane evolution: A new index of lipid peroxidation. Science, 183, 208-210.

12. Asakawa, T., and Matsushita, S. (1980): Coloring condition of thiobarbituric acid test for detecting lipid hydroperoxides. Lipids, 15, 137-140.

13. Bunyan, J., Green, J., Edwin, E.E., and Diplock, A.T. (1960): Studies on vitamin E: Lipid peroxidation in dialuric acid-induced haemolysis of vitamin E-deficient erythrocytes. Biochem. J., 77, 47-51.

14. Hafeman, D.G., and Hoekstra, W.G. (1977): Protection against carbon tetrachloride-induced lipid peroxidation in the rat by dietary vitamin E, selenium, and methionine as measured by ethane evolution. $J$. Nutr., 107, 656-665.

15. Llopis, J., Lampreabe, A., Peran, F., Mataix, F.J., Urbano, G., and Montellano, M.A. (1989): Influence of hydrocortisone acetate administered to the lactating rat on lipid composition of the milk and serum lipid levels in dams and pups. Horm. Metabol. Res., 21, 421-426.

16. Dumelin, E.E., and Tappel, A.L. (1977): Hydrocarbon gases produced during in vitro peroxidation of polyunsaturated fatty acids and decomposition of preformed hydroperoxides. Lipid, 12, 894-900.

17. Hien, P.V., Kovacs, K., and Matkovics, B. (1974): Properties of enzymes. I. Study of superoxide dismutase activity change in human placenta of different ages. Enzyme, 18, 341347.

18. Sekiba, K., and Yoshioka, T. (1977): Lipid peroxidation and SOD activity in the human placental tissue. Acta Obst. Gynaecol. Jpn., 29, 1688-1692 (in Japanese).

19. Igarashi, T., Satoh, T., Ueno, K., and Kitagawa, H. (1983): Sex-related difference in the hepatic glutathione level and related enzyme activities in rat. J. Biochem., 93, 33-36. 